\title{
Twist-projected two-dimensional acoustic topological insulators
}

\author{
Ming-Jun Tuo, ${ }^{1}$ Li-Heng Zhang, ${ }^{1}$ Dong Liu, ${ }^{1}$ Ru-Wen Peng,,${ }^{1, *}$ Ren-Hao Fan, ${ }^{1}$ Ze-Guo Chen, ${ }^{2}$ Ying Wu, \\ Dong-Xiang Qi, ${ }^{1}$ and Mu Wang ${ }^{1, \dagger}$ \\ ${ }^{1}$ National Laboratory of Solid State Microstructures, School of Physics, and Collaborative Innovation Center of Advanced \\ Microstructures, Nanjing University, Nanjing 210093, China \\ ${ }^{2}$ Division of Computer, Electrical and Mathematical Sciences and Engineering, King Abdullah University of Science and Technology, \\ Thuwal 23955-6900, Saudi Arabia
}

(Received 10 January 2019; published 24 May 2019)

\begin{abstract}
Acoustic analogs of electronic or photonic topological insulators provide unique approaches to manipulate sound wave propagation. Inspired by twist-induced topological photonic insulators, here we propose a type of two-dimensional acoustic topological insulator (TI) via projecting a section of a three-dimensional twisting structure to a plane, assembling the projected meta-atoms into metamolecules, and arranging the metamolecules into unit cells to form a honeycomb lattice. It follows that in this acoustic TI, topological phases mimic pseudospin-up and pseudospin-down states, and the pseudospin-orbital couplings are tuned via changing the rotation angles of the meta-atoms, which eventually leads to band inversion. By calculating acoustic band structures, pressure field distributions, and spin Chern numbers of bands, we verify that the topological phase transition occurs around the double Dirac cone and present the topological phase diagram as a function of the rotation angle of the meta-atoms. Once the coupling between adjacent metamolecules is sufficiently strong, mode inversion of topological states emerges. Furthermore, we numerically demonstrate the existence of topologically protected edge states. It is shown that robust pseudospin-dependent one-way transmission is immune to defects at the edge of topological distinct regions, which can be applied to acoustic wave transmissions and communications. Our approach in acoustic systems provides a strategy to explore abundant topological states in two-dimensional systems.
\end{abstract}

DOI: 10.1103/PhysRevB.99.205432

\section{INTRODUCTION}

Topology in mathematics describes the property of space preserved in continuous deformation, which plays an important role in a number of phenomena in condensed-matter physics, such as the quantum Hall effect (QHE) [1,2], quantum spin Hall effect (QSHE) [3,4], topological insulators (TIs) [5-7], etc. In the past decades, topological states have been extensively investigated in photonics [8-17], phononics [18-31], and mechanics [32-34] and have led to some unique wave transport effects. In acoustics, however, conventional media (such as air, water, etc.) support only longitudinal vibration of sound polarization, which cannot generate a spinlike degree of freedom for the acoustic QHE. So far several efforts have been devoted to mimicking the QHE by introducing circulating flow fields [25,26] or employing angular-momentum bias [27]. Similarly, an analog of the Floquet topological insulator has been proposed in the coupled ring resonator waveguide [28-30]. However, it remains challenging for applications due to inevitable noises, dynamic instabilities, complicated fabrication processes, etc. Very recently, artificial phononic "graphene" was introduced to achieve "acoustic topological insulators" [20-22], where "pseudospin" and "pseudospinorbital interaction" were realized without applying external

\footnotetext{
*Corresponding author: rwpeng@nju.edu.cn
}

${ }^{\dagger}$ Corresponding author: muwang@nju.edu.cn fields. And the acoustic topological phase transition was observed when band inversion occurs [18-22]. These significant developments imply possible acoustic analogs of electronic/photonic topological insulators, which can be applied in guiding acoustic waves.

On the other hand, twist is a mathematical concept representing the torsion of a curve in space. In fact, twisting can be considered an operation leading to structural change of materials in three dimensions and has been applied to design threedimensional (3D) elastic chiral metamaterials [35] as well as helical waveguides for light [36,37] and sound [38]. Usually, in $3 \mathrm{D}$ photonic or phononic topological crystals [39-44], a twist is introduced to break the inversion symmetry in order to achieve Weyl points. For example, in Ref. [39], a twist induces chiral interlayer coupling, thus creating an effective acoustic gauge flux closely related to Weyl points in the 3D band structure. In this way, a synthetic gauge potential is induced, and a geometric phase arises, which lead to helical transport of waves associated with topological properties. In addition, a photonic Floquet topological insulator can also be realized by twisted helical waveguides arranged in a graphenelike honeycomb lattice [45].

In this work, we propose a unique type of two-dimensional (2D) acoustic TIs by projecting a 3D twisted structure to a plane. More specifically, when a section of a 3D helical wire bundle is projected onto a plane, the projected meta-atoms are assembled into a metamolecule, and the metamolecule is used as a unit cell to form a honeycomb lattice. In such a 
2D structure, we find the meta-atoms have different rotation angles in the metamolecules. By calculating the acoustic band structure, pressure field distributions, and spin Chern numbers of bands, we verify the topological phase transition around the double Dirac cone in the metastructures. It is shown that the topological phases mimic both pseudospin-up and pseudospin-down states, and the pseudospin-orbital coupling can be tuned with different rotation angles of meta-atoms in the metamolecules. Once the coupling between adjacent metamolecules becomes strong enough, mode inversion takes place, resulting in the topological phase transition, which is affirmed by the change in the spin Chern numbers of the bands. Furthermore, we numerically demonstrate the existence of topologically protected edge states. We suggest that our approach provides a unique way to explore abundant topological states in 2D systems.

This paper is organized as follows. After the introduction, in Sec. II we describe the method to construct a 2D acoustic TI by projecting a 3D twisted structure. In Sec. III, based on the wave equation of sound, the acoustic band structures of the TIs are calculated by using the finite-element method, followed by an analysis of the pseudospin states. We also show that the pseudospin-orbital couplings are tuned by different rotation angles of meta-atoms. Mode inversion of topological states is also demonstrated. In addition, the topological phase transition is confirmed by the change in the spin Chern numbers of the bands. In Sec. IV, we present a topological phase diagram as a function of the rotation angle of the meta-atom, which is simplified to an ellipse, and illustrate the influence of the ellipticity of the meta-atom on the band gap. In Sec. V, we provide several examples to show the topological edge states and the robust pseudospin-dependent one-way transmission. Finally, we summarize our results in Sec. VI.

\section{TWO-DIMENSIONAL ACOUSTIC TOPOLOGICAL INSULATORS FROM THE PROJECTION OF 3D TWISTED STRUCTURES}

Let us start from a 3D twisted structure, consisting of a bundle of six helical wires in one pitch [as shown in Fig. 1(a)]. Each helical wire can be described by a group of Cartesian coordinates $[(x(t), y(t), z(t))]$, which follows parametric equations as

$$
\begin{aligned}
& x(t)=R_{0} \cos \left(\theta_{0}\right)+r_{0} \cos \left(\Omega t+\theta_{0}\right), \\
& y(t)=R_{0} \sin \left(\theta_{0}\right)+r_{0} \sin \left(\Omega t+\theta_{0}\right), \\
& z(t)=t,
\end{aligned}
$$

where $t$ stands for the position of the helical wire along the $z$ axis, $r_{0}$ is the helical radius, and $R_{0}$ is the distance between the center of the bundle and the center of the helical wire. $\Omega=2 \pi / z_{0}$ is the frequency of rotation $\left(z_{0}\right.$ is the periodicity), $\theta_{0}=\pi / 3 \times i(i=0,1,2, \ldots, 5)$ represents the initial phase of the helical wire, and the difference between the adjacent ones is $\pi / 3$ in succession in order to keep the $C_{6}$ symmetry of the wire bundle.

Now we try to reduce this $3 \mathrm{D}$ twisted structure to a $2 \mathrm{D}$ plane as follows. First, by projecting the helical wire bundle with a finite height to the $x y$ plane, we obtain six metaatoms possessing $C_{6}$ symmetry [Fig. 1(a)]. This assembly of (a)
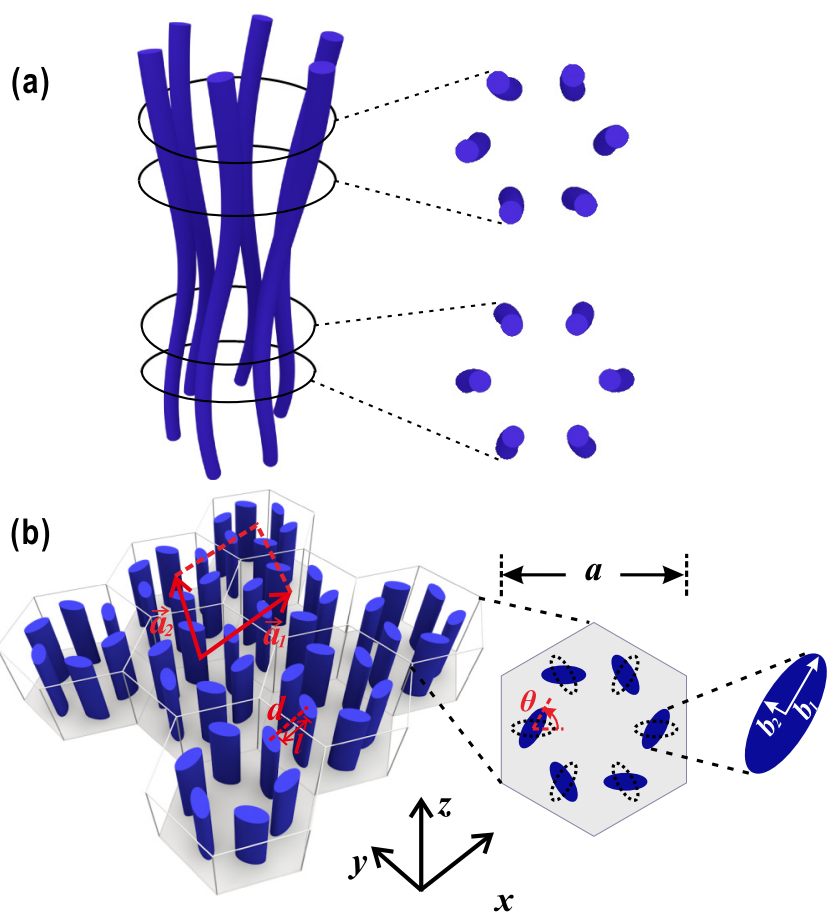

FIG. 1. (a) Schematic of the twisted wire bundle and its projections to $x y$ plane. By projecting a bundle of six helical wires with a finite height to the $x y$ plane, we obtain six meta-atoms with $C_{6}$ symmetry, which are assembled into a metamolecule. Obviously, the rotation angle $\theta$ of meta-atoms changes in the metamolecule if the center of the projected wire section moves on the $z$ axis, as marked in the plot. For example, the rotation angles of meta-atoms become distinct in two different regions. (b) Schematic of the metamolecules assembled into a honeycomb lattice. The meta-atoms shown in (a) are simplified to the elliptical shapes, and the metamolecule with six meta-atoms is used as a unit cell in a $2 \mathrm{D}$ honeycomb lattice. The lattice constant is $a$ with unit vectors $\vec{a}_{1}$ and $\vec{a}_{2}$. The separation between centers of neighboring meta-atoms in the two adjacent metamolecules is $d=a / 3$, and the nearest separation between these two meta-atoms along the $x$ axis is $l$. The inset shows an enlarged view of the metamolecule and the meta-atom, where $\theta$ is the rotation angle of the meta-atoms in the metamolecule. The semimajor axis and the semiminor axis are $b_{1}$ and $b_{2}$.

six meta-atoms is regarded as a metamolecule. In order to describe the metamolecules projected from different heights, we consider a helical wire with $\theta_{0}=0^{\circ}$ (i.e., the center of the helical wire locates on the $x$ axis) and define the rotation angle $\theta$ of the meta-atom as the intersection angle between the $x$ axis and its own principal axis, which satisfies $\tan \theta=d y / d x$ at the central point of the corresponding finite-height helical wire projected onto the plane; thus, the rotation angle of the meta-atom can be written as $\theta=\Omega t+\pi / 2$. Clearly, the rotation angle of meta-atoms changes continuously as it goes along the $z$ axis. As shown in Fig. 1(a), we mark two different regions where the rotation angles of meta-atoms are clearly distinct.

The projected meta-atom does not exhibit a regular shape, which makes later analysis difficult. Second, we simplified each meta-atom to an ellipse with the ellipticity defined as $\gamma=b_{1} / b_{2}$, where $b_{1}$ is the semimajor axis and $b_{2}$ is the 
(a)

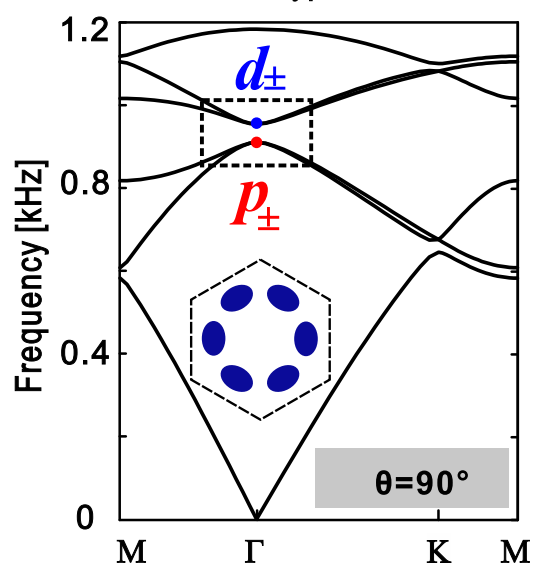

(b)

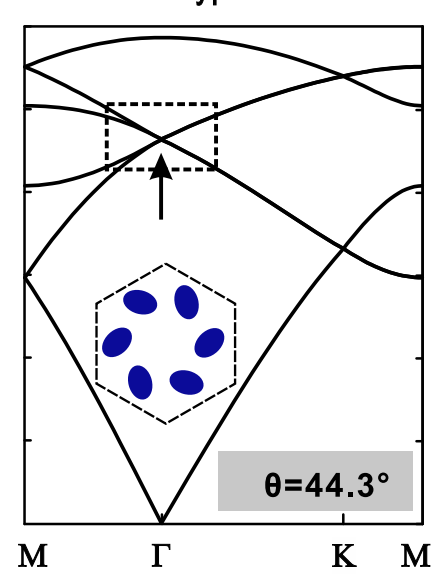

(c)

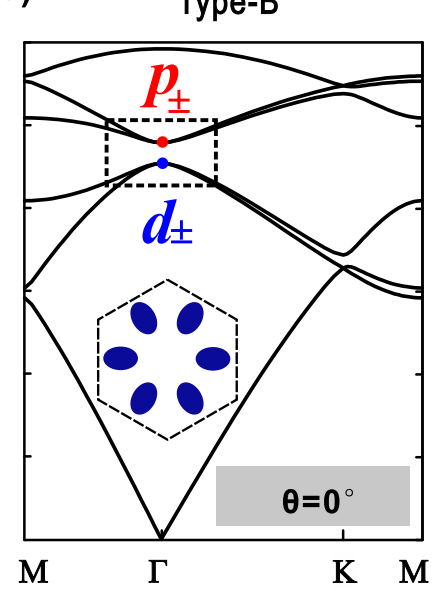

FIG. 2. The calculated acoustic band structure of the 2D metasystems with different rotation angles $\theta$ of meta-atoms. (a) The band structure of the metastructure type A $\left(\theta=90^{\circ}\right)$, where a trivial band gap occurs at the $\Gamma$ point. (b) The band structure of the metastructure type $\mathrm{C}$ $\left(\theta=44.3^{\circ}\right)$, where a double Dirac cone emerges at the $\Gamma$ point. (c) The band structure of the metastructure type B $\left(\theta=0^{\circ}\right)$, where an inverted nontrivial band gap appears at the $\Gamma$ point. The inset shows the corresponding unit cell and the state occupation below and above the gap at the $\Gamma$ point. In all three metastructures, the air meta-atoms embed in a water background, and the geometrical parameters are taken to be $a=3 \mathrm{~cm}, b_{1}=0.35 \mathrm{~cm}, b_{2}=0.23 \mathrm{~cm}$.

semiminor axis. Such simplification does not change the $C_{6}$ symmetry, so the essential physics is preserved. Third, the metamolecule with six meta-atoms is assembled as a unit cell in a 2D honeycomb lattice [Fig. 1(b)], where the lattice constant is $a$. It will be verified in Sec. III that we eventually construct a twist-projected 2D acoustic TI. The separation between the centers of the neighboring meta-atoms in the adjacent metamolecules is $d=a / 3$, and the nearest separation of these two meta-atoms along the $x$ axis is $l=d-$ $2 \sqrt{b_{1}^{2} \cos ^{2} \theta+b_{2}^{2} \sin ^{2} \theta}$. Then, we define a separation ratio $\eta=l / d$ to describe the nearest interaction of the neighboring metamolecules. By decreasing the separation ratio $\eta$, the coupling between adjacent metamolecules becomes stronger and eventually leads to the topological phase transition from the topological trivial state to the nontrivial state.

\section{THE ACOUSTIC BAND STRUCTURES, THE PSEUDOSPIN STATES AND THEIR SPIN CHERN NUMBERS, AND THE BAND INVERSION}

The intrinsic difference between a phonon and an electron is that the phonon does not have a spinlike degree of freedom, whereas spin is essential for the QSHE. Following the scheme in Sec. II, we can construct an acoustic Kramers doublet to mimic electrons. Our scheme requires the resonators possess a higher refractive index than the ambient medium, such as perforated air holes in polished stainless-steel plates [20], aerogel as a soft acoustic metamaterial in air [46], and air in water [21]. Without loss of generality, we choose the air-inwater system, where the air is capsulated by water. Such a system can be achieved by several experimental approaches [47-49]. For example, one may use ultrasound contrast agent microbubble suspension [47] or bubble rafts in water [48]. The propagation of sound in this system satisfies the wave equation [50]:

$$
\nabla \cdot\left(\frac{1}{\rho(\vec{r})} \nabla P(\vec{r})\right)=-\frac{\omega^{2}}{v(\vec{r})^{2}} \frac{P(\vec{r})}{\rho(\vec{r})},
$$

where $P$ is the sound pressure and $\omega$ is the angular frequency of its oscillation. Here, $\rho$ and $v$ are the mass density and longitudinal sound speed, respectively. Based on Eq. (2), we carry out numerical calculations of the acoustic band structures of several different metastructures with a commercial software package (COMSOL MULTIPHYSICS). In the calculations, the continuity condition of both sound pressure and the normal velocity of water/air are applied at the boundary between water and air. Periodic boundary conditions are applied in the system to calculate the band structure, but to simulate the edge states in a finite system, the plane wave radiation condition is applied at the edge of system. The mass density and longitudinal sound speed used in the calculations are $\rho_{0}=1000 \mathrm{~kg} / \mathrm{m}^{3}$ and $v_{0}=1490 \mathrm{~m} / \mathrm{s}$ for water and $\rho_{1}=$ $1.25 \mathrm{~kg} / \mathrm{m}^{3}$ and $v_{1}=343 \mathrm{~m} / \mathrm{s}$ for air.

We consider three types of unit cells with different rotation angles $\theta$, which are denoted as type $\mathrm{A}$, with $\theta=90^{\circ}$; type $\mathrm{C}$, with $\theta=44.3^{\circ}$; and type $\mathrm{B}$, with $\theta=0^{\circ}$. The corresponding acoustic band structures are illustrated in Figs. 2(a)-2(c), where type $\mathrm{C}$ possesses a double Dirac cone. In fact types $\mathrm{A}$ and $\mathrm{B}$ represent two distinct scenarios with maximum and minimum values of the separation ratio $\eta$, respectively, both of which maintain $C_{6}$ symmetry. The $C_{6}$ symmetry group has two irreducible representations, i.e., $E_{1}$ and $E_{2}$, whose base functions are $x(y)$ and $x y\left(x^{2}-y^{2}\right)$, corresponding to odd and even parities, respectively [51]. The irreducible representations of the rotation operators are given by

$$
D_{E m}\left(C_{6}\right)=\left(\begin{array}{cc}
\cos \varphi & -\sin \varphi \\
\sin \varphi & \cos \varphi
\end{array}\right)
$$


where $m=1,2$, with $\varphi=\pi / 3 \times m$ for $D_{E m}\left(C_{6}\right)$ on the bases $(x, y)^{T}$ and $\left(x y, x^{2}-y^{2}\right)^{T}$.

Similar to the topological photonic crystal proposed in Ref. [9], we define the pseudo-time-reversal (TR) operator in the present acoustic metastructure, which has the same mathematical form as that in the photonic system (see Ref. [9]), i.e.,

$$
\mathcal{T}=\mathcal{U}\left(D_{E m}\right) \mathcal{K}=-i \sigma_{y} \mathcal{K}
$$

with a unitary operator

$$
\begin{aligned}
\mathcal{U}\left(D_{E m}\right) & =\left[D_{E m}\left(C_{6}\right)-(-1)^{m} D_{E m}\left(C_{6}^{2}\right)\right] / \sqrt{3} \\
& =\left(\begin{array}{cc}
0 & -1 \\
1 & 0
\end{array}\right)=-i \sigma_{y} .
\end{aligned}
$$

Here, $\sigma_{y}$ is the Pauli matrix, and $\mathcal{K}$ is the complex conjugate operator. It can be verified that $\mathcal{T}^{2}=-1$; thus, the present metastructure possesses the pseudo-TR symmetry in acoustics, where Kramers doubling is guaranteed in the same way as the TR symmetry in the electronic systems and also the pseudo-TR symmetry in the photonic systems [9].

As shown in Figs. 2-4, the Kramers doublets in the band dispersions appear at the $\Gamma$ point in the form of two doubly degenerate states (pseudospin up and pseudospin down) due to the $C_{6}$ crystalline symmetry. These acoustic metamolecules carry the $p_{x}\left(p_{y}\right)$ and $d_{x y}\left(d_{x^{2}-y^{2}}\right)$ orbitals, which possess the same symmetry as electronic orbitals of conventional atoms in solids and also the characteristic of pseudospin similar to that of a topological photonic crystal. The two pseudospin states are given by $p_{ \pm}=\left(p_{x} \pm i p_{y}\right) / \sqrt{2}$ and $d_{ \pm}=\left(d_{x^{2}-y^{2}} \pm\right.$ $\left.i d_{x y}\right) / \sqrt{2}$, which are related to the above-mentioned base functions $x(y)$ and $x y\left(x^{2}-y^{2}\right)$ for these two irreducible representations, i.e., $E_{1}$ and $E_{2}$, respectively. This means that the pseudospin-up and pseudospin-down states correspond to the positive and negative angular momenta of the pressure fields, respectively. For the scenario of type A $\left(\theta=90^{\circ}\right)$, as shown in Fig. 2(a), the band below (above) the gap is occupied by $p_{ \pm}\left(d_{ \pm}\right)$, and the separation ratio $\eta$ reaches its maximum. As the rotation angle $\theta$ of the meta-atoms gradually decreases, the band gap narrows accordingly. When the rotation angle reaches $\theta=44.3^{\circ}$ [type C, as shown in Fig. 2(b)], the $p_{ \pm}$and $d_{ \pm}$states degenerate at the $\Gamma$ point, the gap disappears, and an accidental double Dirac cone is generated. By continuously decreasing the rotation angle $\theta$ of the meta-atoms, the band gap reopens. For the scenario of type $\mathrm{B}\left(\theta=0^{\circ}\right)$, as shown in Fig. 2(c), the state occupation at the lower (higher) frequency of the gap exhibits $d_{ \pm}\left(p_{ \pm}\right)$characters at the $\Gamma$ point, and the separation ratio $\eta$ reaches its minimum, opposite the scenario of type A. Namely, band inversion takes place upon rotating the metamolecule in the system.

More specifically, we illustrate the pressure field distributions at the $\Gamma$ point in several metastructures, as shown in Figs. 3(a)-3(d). For the scenario of type A, the two doubly degenerate acoustic states, which are denoted by $d_{x y}\left(d_{x^{2}-y^{2}}\right)$ and $p_{x}\left(p_{y}\right)$ orbitals, appear at higher [Fig. 3(a)] and lower [Fig. 3(c)] frequencies of the band gap in Fig. 2(a), respectively. For the scenario of type B, two inverted doubly degenerate acoustic states appear at higher [Fig. 3(b)] and lower [Fig. 3(d)] frequencies of the band gap in Fig. 2(c), which (a)

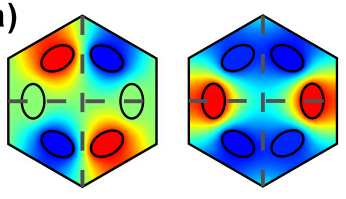

(b)
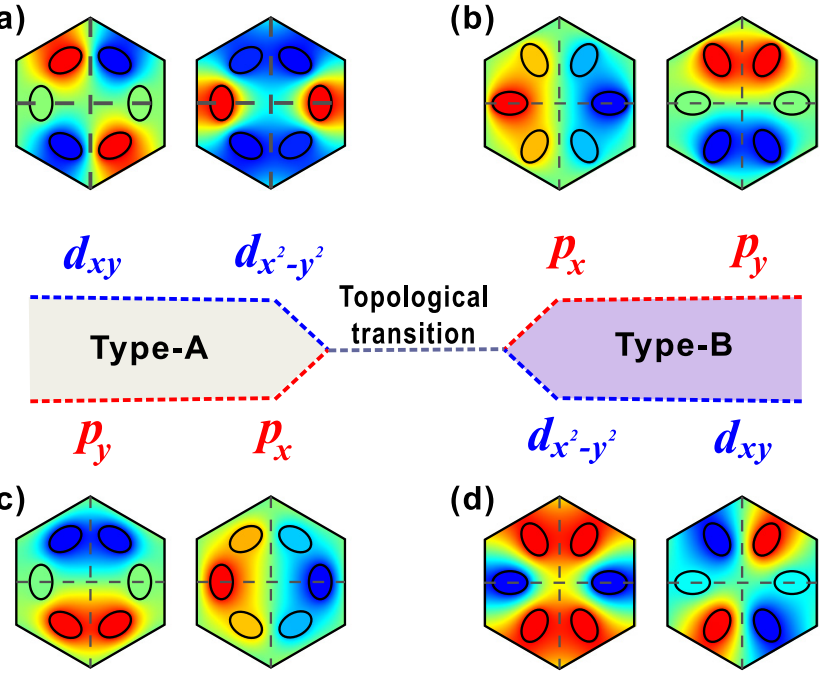

FIG. 3. The pressure field distributions at the $\Gamma$ point are shown for scenarios of type A and type B in Figs. 2(a) and 2(c). In all cases, the pressure field possesses $p_{x}\left(p_{y}\right)$ and $d_{x y}\left(d_{x^{2}-y^{2}}\right)$ orbitals, which are separated by a bulk band gap. For the scenario of type A (left, with $\theta=90^{\circ}$ ), the two doubly degenerate acoustic states denoted by $d_{x y}\left(d_{x^{2}-y^{2}}\right)$ and $p_{x}\left(p_{y}\right)$ orbitals appear at (a) the higher frequency and (c) the lower frequency, respectively; for the scenario of type B (right, with $\theta=0^{\circ}$ ), two inverted doubly degenerate acoustic states appear at (b) higher frequency and (d) lower frequency, corresponding to $p_{x}\left(p_{y}\right)$ and $d_{x y}\left(d_{x^{2}-y^{2}}\right)$ orbitals, respectively. This feature demonstrates clearly the mode inversion in the system.

correspond to $p_{x}\left(p_{y}\right)$ and $d_{x y}\left(d_{x^{2}-y^{2}}\right)$ orbitals, respectively. Clearly, we demonstrate the mode inversion in the metastructures.

To further verify the pseudospin states, we also calculate the real-space distributions of the time-averaged acoustic energy flow $\overrightarrow{\boldsymbol{I}}=\frac{1}{T} \int_{0}^{T} \operatorname{Re}(p) \operatorname{Re}(\overrightarrow{\boldsymbol{v}}) d t$ at $T=2 \pi / \omega$ in the metamolecule, as illustrated in Figs. 4(a)-4(d) for both types $\mathrm{A}$ and $\mathrm{B}$. The energy flow circulates around the center of metamolecule, and different chiralities of the energy flow represent different pseudospin states. For the scenario of type A, the pseudospin states denoted by the pressure field with positive and negative angular momenta $d_{ \pm}=\left(d_{x^{2}-y^{2}} \pm i d_{x y}\right)$ and $p_{ \pm}=\left(p_{x} \pm i p_{y}\right)$ appear at higher [Fig. 4(a)] and lower [Fig. 4(c)] frequencies, respectively. The energy flows around the individual molecules at the lower frequency [Fig. 4(c)]. For the scenario of type $\mathrm{B}$, the $p_{ \pm}$and $d_{ \pm}$states occupy higher [Fig. 4(b)] and lower [Fig. 4(d)] frequencies, respectively, implying the band inversion. In addition, by comparing Fig. 4(d) with Fig. 4(c), we can find that the energy flow in type $\mathrm{B}$ is enhanced in the interstitial regimes.

The pseudospin channels can be characterized by the spin Chern numbers [9,18]. Zero-spin Chern numbers indicate topological trivial phases, while nonzero-spin Chern numbers infer topological nontrivial phases. Following the method given in Ref. [52], we have numerically calculated Berry curvatures and the spin Chern numbers of the pseudospin states $p_{ \pm}$and $d_{ \pm}$. Starting from the simulated pressure field distributions as a function of wave vector $\vec{k}$ in the metastructures, we have calculated the Berry connection $B(\vec{k})$ 
(a)
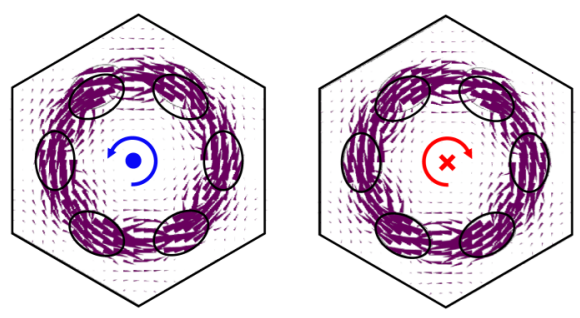

$d_{+}$

d. (b)

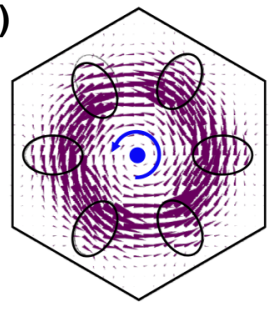

$p_{+}$

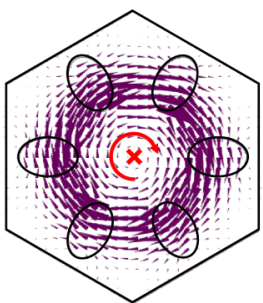

p

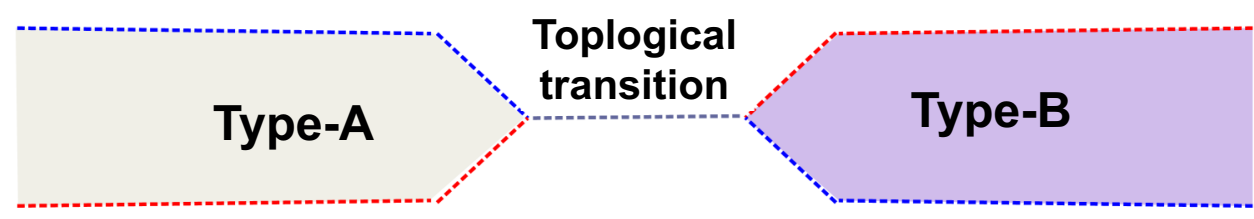

$p$

(c)

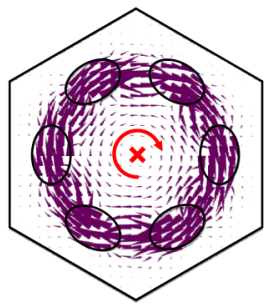

$p_{+}$

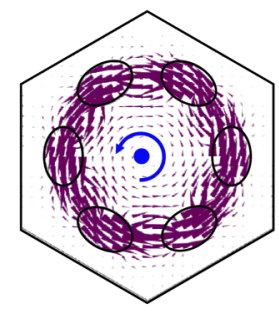

d.

(d)

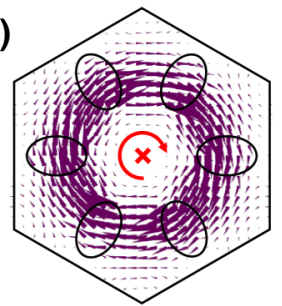

$d_{+}$

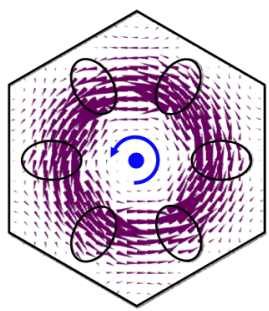

FIG. 4. The acoustic energy flow at the $\Gamma$ point for type A and type B in Figs. 2(a) and 2(c). For the scenario of type A (left, with $\left.\theta=90^{\circ}\right)$, the pseudospin states denoted by the pressure field distributions with positive and negative angular momenta $d_{ \pm}=\left(d_{x^{2}-y^{2}} \pm i d_{x y}\right)$ and $p_{ \pm}=\left(p_{x} \pm i p_{y}\right)$ appear at (a) the higher frequency and (c) the lower frequency, respectively. For the scenario of type B (right, with $\left.\theta=0^{\circ}\right)$, the pseudospin states denoted by the pressure field distributions with $p_{ \pm}=\left(p_{x} \pm i p_{y}\right)$ and $d_{ \pm}=\left(d_{x^{2}-y^{2}} \pm i d_{x y}\right)$ appear at $(\mathrm{b})$ the higher frequency and (d) the lower frequency, respectively.

and the Berry curvature $\Omega(\vec{k})=\nabla \times B(\vec{k})$. Figures $5($ a) and 5 (b) illustrate the calculated Berry curvature $\Omega(\vec{k})$ of the $p_{ \pm}$ and $d_{ \pm}$states around the $\Gamma$ point in the metastructure for types $\mathrm{A}$ and $\mathrm{B}$, respectively. By integrating the Berry curvature over the first Brillouin zone, the spin Chern numbers $C_{s}=$ $\frac{1}{2 \pi} \int_{F B Z} \Omega(\vec{k}) d^{2} \vec{k}$ for these states are achieved. From Fig. 5, it is found that $C_{s}=0$ for these $p_{ \pm}$and $d_{ \pm}$states in type A, which indicates topological trivial phases; however, in type $\mathrm{B}, C_{s}=+1$ for the pseudospin-up states $p_{+}$and $d_{+}$, but $C_{s}=-1$ for the pseudospin-down states $p_{-}$and $d_{-}$, which indicates the topological nontrivial phases. These numerically calculated spin Chern numbers are the same as the analytical results from $\mathbf{k} \cdot \mathbf{P}$ theory $[9,18]$, which starts from the effective Hamiltonian.

By revisiting Figs. 2-4, we can find that for the scenario of type $\mathrm{A}\left(\theta=90^{\circ}\right)$, the topological trivial states $p_{ \pm}\left(d_{ \pm}\right)$occupy the band below (above) the gap, making it a conventional insulator. This is verified by the zero-spin Chern number. By decreasing the rotation angle $\theta$ of the meta-atoms, the band gap becomes narrower. Once the rotation angle reaches $\theta=44.3^{\circ}$ (type $\mathrm{C}$ ), states $p_{ \pm}$and $d_{ \pm}$degenerate at the $\Gamma$ point, the gap disappears, and an accidental double Dirac cone appears, signifying the occurrence of the topological phase transition. By further decreasing $\theta$, the band gap reopens. For the scenario of type B $\left(\theta=0^{\circ}\right)$, the topological nontrivial states $p_{ \pm}\left(d_{ \pm}\right)$occupy the band above (below) the gap, which is opposite the scenario of type A and suggests the existence of an unconventional insulating state. This is verified by the nonzero-spin Chern number. Therefore, band inversion does occur together with the topological phase transition, which is affirmed by the change in the spin Chern numbers of the bands.

\section{TOPOLOGICAL PHASE DIAGRAM}

For a better understanding of the topological evolution in our system, we calculate the acoustic band structures of the metastructures as a function of rotation angle $\theta$ of meta-atoms. Here we focus on the band gap around the double Dirac cone. As shown in Fig. 6(a), the red and blue curves represent the modes corresponding to the $p_{ \pm}$and $d_{ \pm}$states, respectively. The separation between these two modes corresponds to the band gap. It is obvious that the band gap is symmetric with respect to $\theta=0^{\circ}$ due to the mirror symmetry of the unit cell, and the local maxima of the band gaps appear at $\theta=90^{\circ}$ (type A) and $\theta=0^{\circ}$ (type $\mathrm{B}$ ). The $p_{ \pm}$and $d_{ \pm}$states at the $\Gamma$ point reverse around the scenario of type $\mathrm{C}$, leading to band inversion in the acoustic band structure of this TI.

We then investigate the influence of the ellipticity $\gamma$ of the elliptic meta-atom on the acoustic band gaps while keeping the filling factor constant. When the double Dirac cone emerges, the rotation angle $\theta$ of meta-atoms in the metamolecule changes almost linearly with the ellipticity $\gamma$ 


\section{(a) "Type-A":}

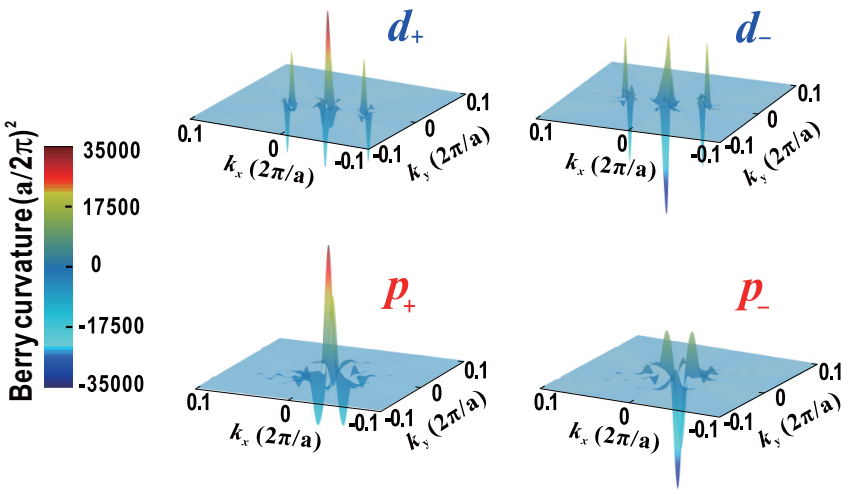

(b) "Type-B":

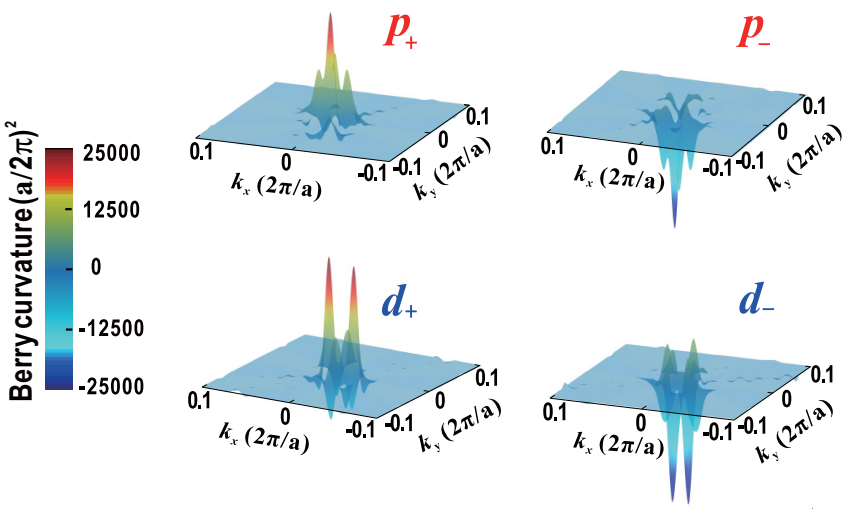

FIG. 5. The calculated Berry curvatures of the pseudospin states $p_{ \pm}$and $d_{ \pm}$around the $\Gamma$ point for (a) type A, and (b) type B. By integrating Berry curvature around the $\Gamma$ point in the first Brillouin zone, we obtain the spin Chern numbers $C_{s}$ for the $p_{ \pm}$and $d_{ \pm}$states. It is shown that $C_{s}=0$ for those $p_{ \pm}$and $d_{ \pm}$states in type A, indicating the topological trivial phase, while $C_{s}=+1$ for both $p_{+}$and $d_{+}$states and $C_{s}=-1$ for both $p_{-}$and $d_{-}$states in type $\mathrm{B}$, indicating the topological nontrivial phase.
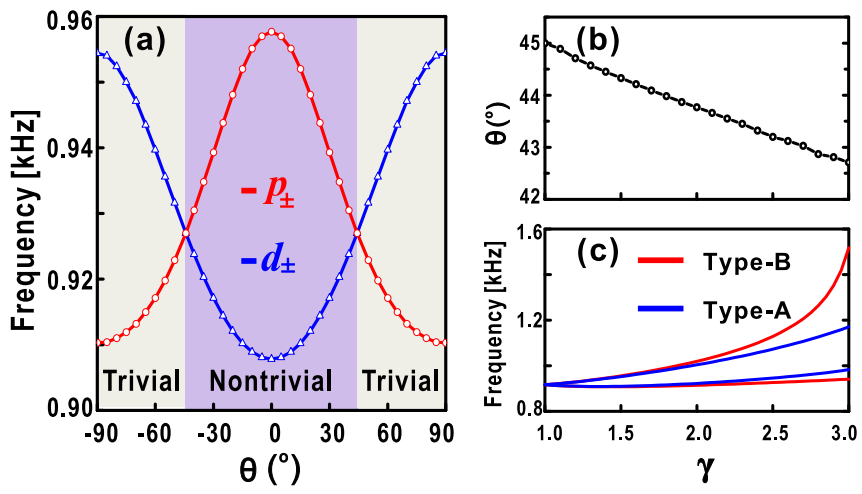

FIG. 6. (a) The topological phase diagram of the metastructures as a function of the rotation angle $\theta$ of the meta-atoms, where the blue (red) curve represents the frequency of the $d_{ \pm}\left(p_{ \pm}\right)$states at the $\Gamma$ point. The trivial and nontrivial regions are marked with different colors. (b) The rotation angle $\theta$ of the meta-atoms corresponding to the double Dirac cone as a function of ellipticity $\gamma$ of the elliptic meta-atom. (c) The frequencies corresponding to the $p_{ \pm}$or $d_{ \pm}$states at the $\Gamma$ point as a function of the ellipticity $\gamma$ of the meta-atom for the scenarios of type $\mathrm{A}\left(\theta=90^{\circ}\right.$, blue curves $)$ and type $\mathrm{B}\left(\theta=0^{\circ}\right.$, red curves). of the elliptic meta-atom [as shown in Fig. 6(b)]. Figure 6(c) presents how the ellipticity $\gamma$ of the meta-atom affects the frequencies corresponding to the $p_{ \pm}$and $d_{ \pm}$states at the $\Gamma$ point for both types A and B, respectively. Obviously for $\gamma=1$, there always exists a double Dirac cone due to symmetry $C_{6 v}$. As the ellipticity $\gamma$ of the meta-atom increases, the band gaps between the $p_{ \pm}$and $d_{ \pm}$states at the $\Gamma$ point significantly grows for both types $\mathrm{A}$ and $\mathrm{B}$, corresponding to the larger maximum and the smaller minimum values of the separation ratio $\eta$, respectively.

\section{THE EDGE STATES AND THE ROBUST PSEUDOSPIN-DEPENDENT ONE-WAY TRANSMISSION}

The most intriguing properties of the acoustic topological insulator are the edge states localized at the interface between the topological trivial and nontrivial regions. We design a ribbon-shaped supercell made of a topological nontrivial region (type B) clad by two topological trivial regions (type A), as illustrated in Fig. 7(a), which prevents possible edge states from leaking into free space. The band gaps of these two different structures have an overlapping frequency regime. The acoustic band structure of the metastructure with the supercells is numerically calculated with periodic boundary conditions in all directions. As shown in Fig. 7(b), a bulk band gap can be found in the range from 900 to $945 \mathrm{~Hz}$. The topological edge modes appear within the bulk band gap, as indicated by the doubly degenerate curves corresponding to two sets of edge states of the ribbon-shaped supercell with opposite group velocities. In fact, there is a tiny gap at the $\Gamma$ point due to the reduction of $C_{6}$ symmetry at the ribbon edge. We further plot the real-space distributions of the pressure field in Fig. 7(c) at typical momenta around the $\Gamma$ point, labeled A and B in Fig. 7(b), with $k_{\|}= \pm 0.04 \times 2 \pi / a$. Two pairs of in-gap edge states are confined at the left and right edges and decay exponentially into the bulk. The energy flow exhibits a nonzero counterclockwise (clockwise) style for the pseudospin-up (pseudospin-down) state at the edge. Thus, in this metastructure, we obtain four pseudospin-dependent transmission channels spatially separated without any external fields. This indicates that counterpropagations of acoustic energy do occur at the same interface associated with two pseudospin states, which is actually the hallmark of a QSHE state.

Based on pseudospin-dependent propagation of edge states in the metastructure, we can design pseudospin-dependent acoustic devices. For example, an acoustic splitter of pseudospins is schematically illustrated in Fig. 8(a). This acoustic splitter contains four sections with the nontrivial purple region (type B) locating in the top left and bottom right sections and the trivial gray region (type A) locating in the top right and bottom left sections. Thus, four edges exist in this splitter, which are marked L (left) and $\mathrm{R}$ (right) along the horizontal direction and $\mathrm{D}$ (down) and $\mathrm{U}$ (up) along the longitudinal one. In order to excite the topological mode, two dipole sources (with phase difference $\pi / 2$ ) are placed at the center of the splitter. More specifically, the pseudospin sources are denoted [18] $S_{ \pm}=P_{0} e^{i \omega t}(\hat{x} \mp i \hat{y})$ in the calculations, where $P_{0}$ is a constant sound pressure, $+(-)$ stands for pseudospin-up (pseudospin-down) source, and $\hat{x}$ and $\hat{y}$ are unit vectors along 

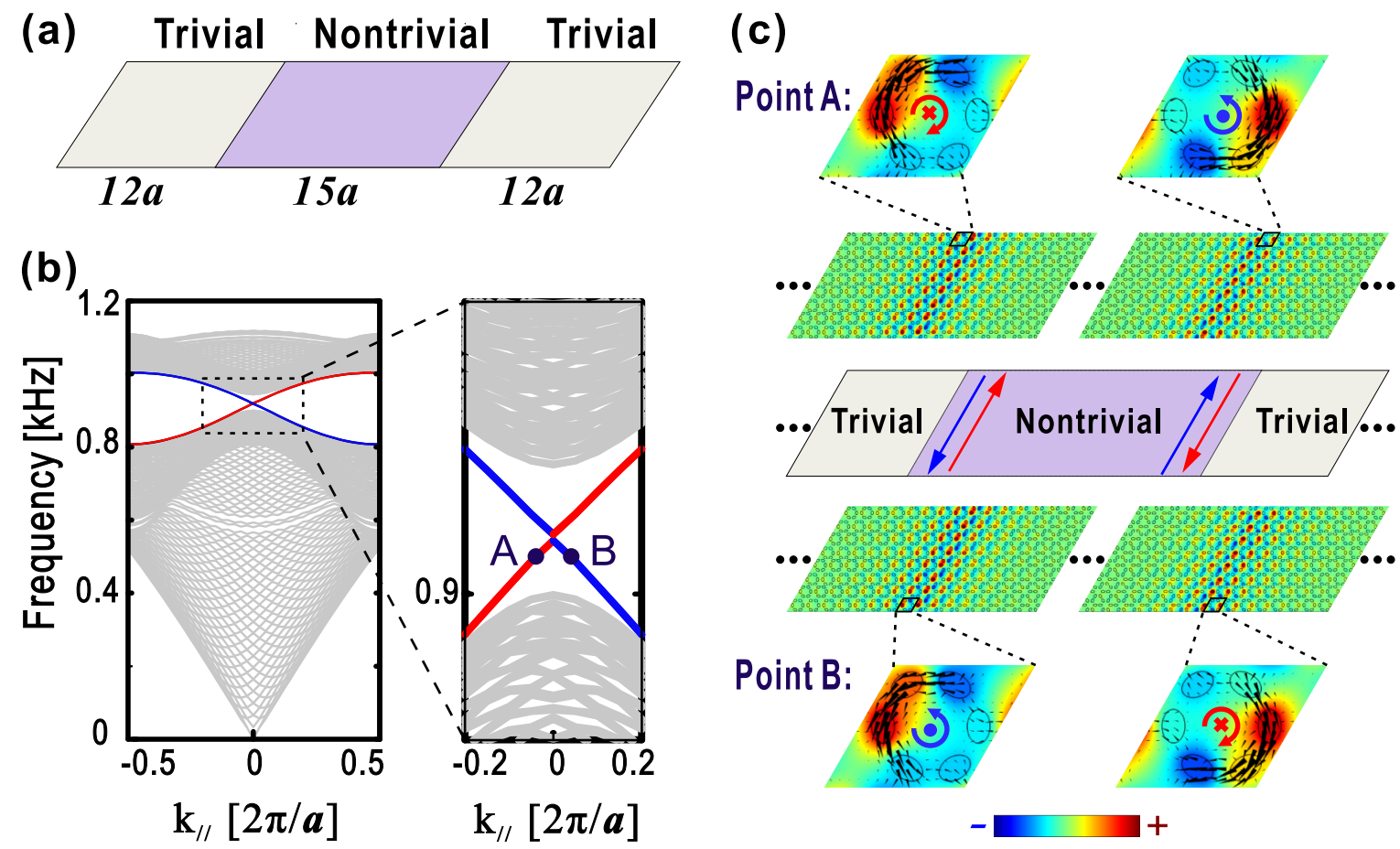

FIG. 7. (a) The schematic of a ribbon-shaped supercell, which is composed of 12 metamolecules for the topological trivial regions (type A) on the left and the right and 15 metamolecules for the topological nontrivial region (type B) in the middle. (b) The acoustic band structure of the metastructure with the ribbon-shaped supercells. The gray regions represent the bulk modes, whereas the doubly degenerate topological edge modes appear in the bulk band gap, as marked by the blue and red curves, respectively. The right panel shows an enlarged view of the acoustic band structure around the bulk band gap. (c) The real-space distributions of the pressure field and the energy flow in the metastructure with the supercells at typical momenta of points A and B in (b), respectively. The rainbow colors represent the amplitude of the pressure field, and the arrows show the direction and amplitude of the energy flow. The upper graph shows the typical momentum of point A in (b); the left (right) edge supports an upward edge mode with pseudospin down (pseudospin up). The lower graph shows the typical momentum of point B in (b); the left (right) edge supports a downward edge mode with pseudospin up (pseudospin down). The middle graph schematically shows four sound transmission channels at the left and right edges, where blue (red) arrows represent the pseudospin up (pseudospin down) and the transport direction of the corresponding pseudospin.

the $x$ and $y$ directions, respectively. In reality, one may combine multiple excitation sources or design specific antennas to mimic such sources. In the following simulation of the edge states, the plane wave radiation condition is applied at the edge of the system. As shown in Fig. 8, when a pseudospin-up source (denoted by the green star) of $920 \mathrm{~Hz}$ is used, acoustic wave propagates along the up and down edges [Fig. 8(b)]; the energy flow of this edge state shows a counterclockwise style, which corresponds to the pseudospin up. But when a pseudospin-down source (denoted by the orange star) of $920 \mathrm{~Hz}$ is used, the acoustic wave propagates along the left and right edges [Fig. 8(c)]; the energy flow of this edge state shows a clockwise style, which corresponds to the pseudospin down. Thus, with this splitter, the transmission channels along the up and down edges open only for pseudospin up, while the transmission channels along the left and right edges open only for pseudospin down.

Furthermore, we show one-way transmission for pseudospins at the edge of the metastructure. For simplicity, we study the propagation of pseudospin down in a metastructure, where the $15 a \times 24 a$ nontrivial region (type B) sits beside the $15 a \times 24 a$ trivial gray region [type A; see the left side of Fig. 8(d)]. If the pseudospin-down source of $920 \mathrm{~Hz}$ (denoted by the orange star) is placed on the bottom of the edge without any defect, the pressure intensity distribution shows clearly that the sound can propagate along the edge without obvious backscattering [see the right side of Fig. 8(d)]. The sound waves are localized in the vicinity of the edge and are excluded from the bulk, indicating that the bulk region is insulating. In contrast to the prohibited transport of pseudospin down along the down edge in Fig. 8(c), the sound propagation shown in Fig. 8(d) indicates the one-way transmission for the pseudospin-down modes.

To demonstrate the robustness of the topological edge states, we further introduce three edges with different types of defects, including meta-atomic disorder, local cavity, and bending [as shown in Figs. 8(e)-8(g)]. We excite the pseudospin-down mode (denoted by the orange star) on the bottom of the edge. The pressure intensity distributions [illustrated in Figs. 8(e)-8(g)] demonstrate unambiguous robust sound transport, which is immune to the backscattering caused by local defects, and the sound waves circumambulate around them. In order to evaluate the robustness, we have further calculated the transmission efficiency of the sound waves propagating along the edge. Figure 9 illustrates the simulated transmission spectra of the sound waves along different edges: (i) without any defects and (ii) with different types of defects, including meta-atomic disorder, local 


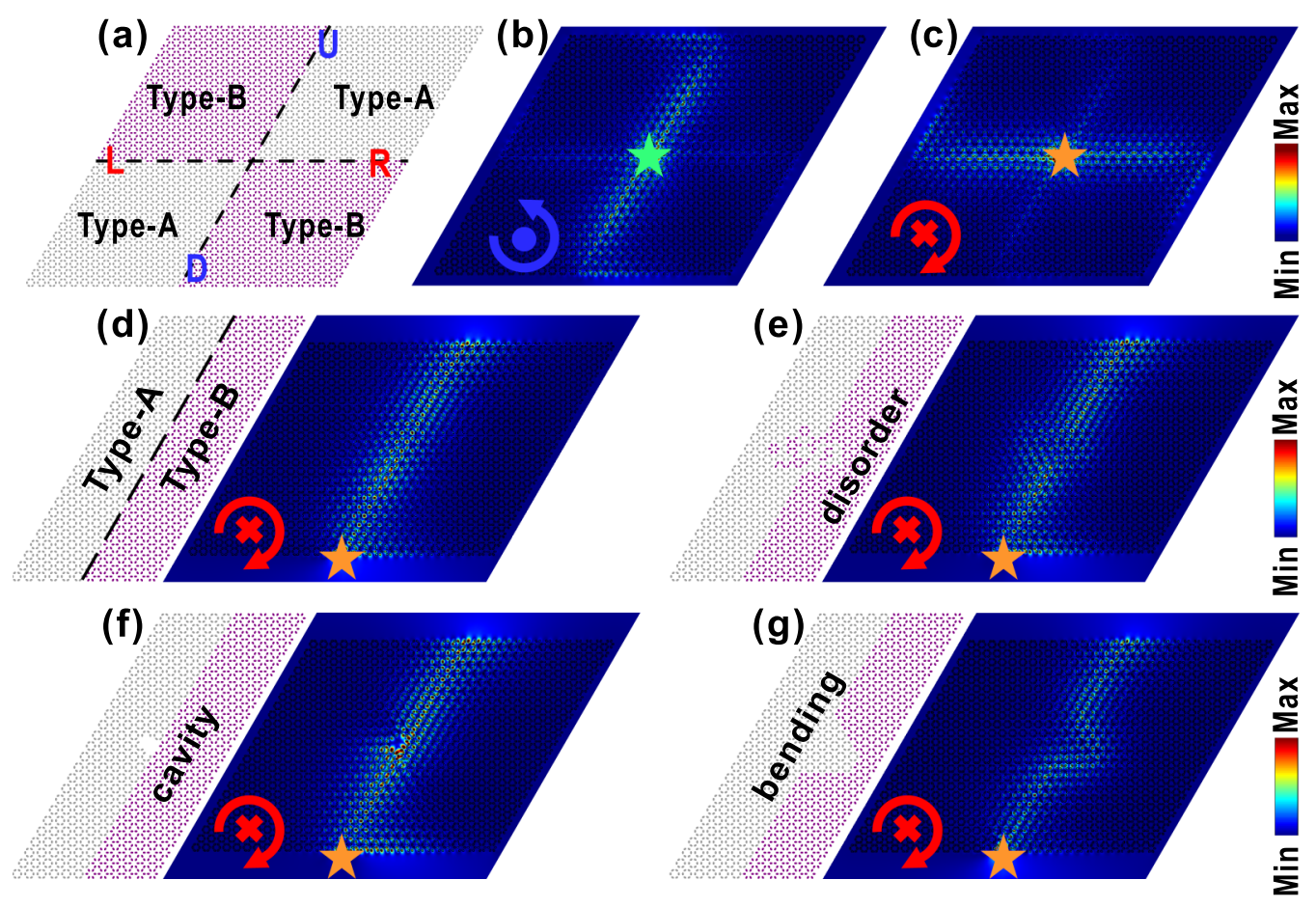

FIG. 8. (a) The schematic of a pseudospin splitter for an acoustic wave. Black dashed lines denote the cross edges in between the topological trivial regions (type A, in gray, $15 a \times 15 a$ for each region) and the topological nontrivial regions (type $\mathrm{B}$, in purple, $15 a \times 15 a$ for each region). There are four edges in total, i.e., the left, right, up, and down edges, which are marked L, R, U, and D, respectively. (b) The calculated pressure intensity distribution when the pseudospin-up source of $920 \mathrm{~Hz}$ is placed at the splitter center. It is shown that the pseudospin-up propagates only along the up and down edges. (c) The calculated pressure intensity distribution when the pseudospin-down source of $920 \mathrm{~Hz}$ is placed at the splitter center. It is shown that the pseudospin-down propagates only along the left and right edges. (d)-(g) The acoustic one-way transmission of pseudospin down and the robustness against defects in the metastructures, with a $15 a \times 24 a$ topological trivial region (type A, gray) beside a $15 a \times 24 a$ topological nontrivial region (type B, purple). The left panels show only the region near the edge between these two regions; the right panels show the calculated pressure intensity distribution when the pseudospin-down source is placed at the bottom of the edge. The metastructure is shown (d) without any defects, (e) with meta-atomic disorder, (f) with local cavity, and (g) with bending. The pressure intensity distributions in (e)-(g) show unambiguous robust sound transport against meta-atomic disorder, local cavity, and bending, respectively. The green (orange) star represents the pseudospin-up (pseudospin-down) source.
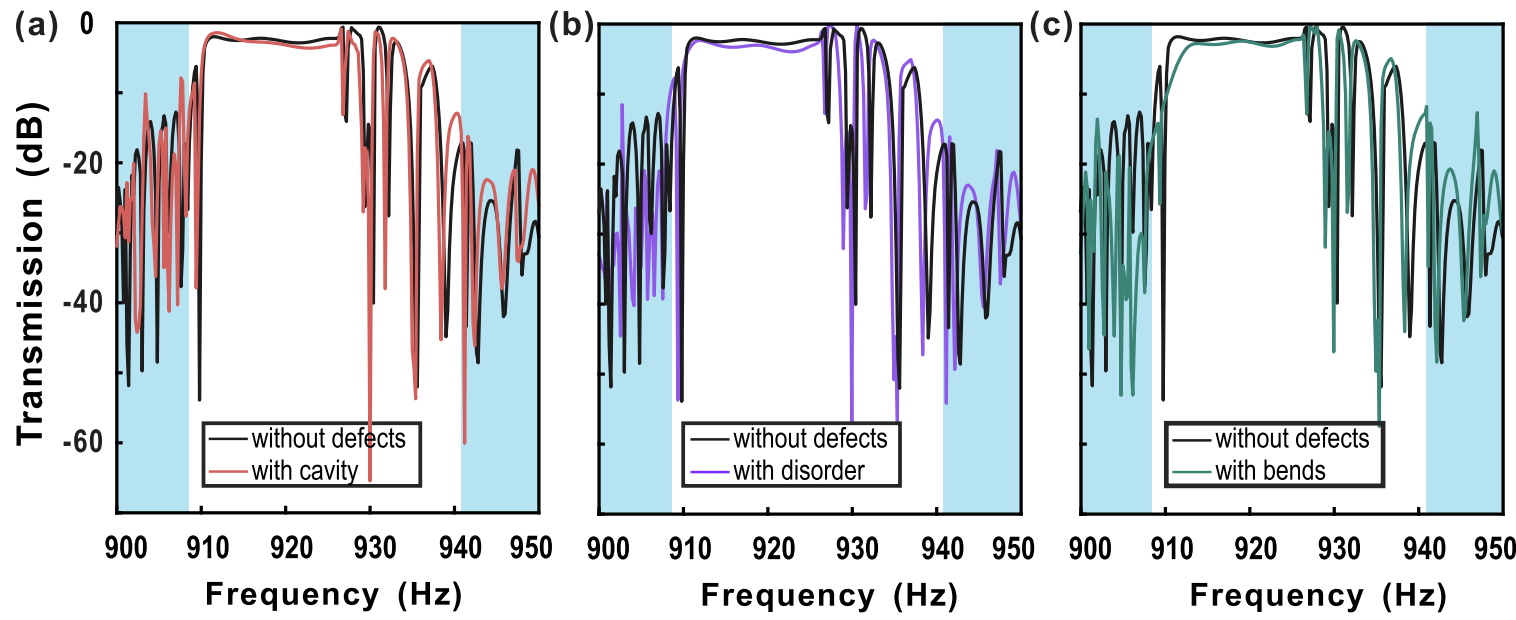

FIG. 9. The simulated transmission spectra of the sound waves along different edges in the metastructures shown in Figs. 8(e)-8(g). The black curves correspond to the scenario without any defects, while (a) the red, (b) blue, and (c) green curves indicate the scenario with local cavity, meta-atomic disorder, and bends, respectively. The shaded regions represent the bulk bands, and the white regions represent the bulk band gaps. It is obvious that the transmissions through the edges are relatively high within the bulk band gap, and they are nearly unaffected by the defects, especially around the center of the band gap. 
cavity, and bending. The shaded regions correspond to the bulk bands, and the white regions correspond to the bulk band gaps. It is obvious that the transmissions through all these edges are relatively high within the bulk band gap, and transmission efficiency through the edges is nearly unaffected by the defects (as shown in Fig. 9). These effects show that pseudospin-dependent topological edge states have intrinsic robust characteristics despite the existence of different types of defects, which is completely different from the scenario of conventional waveguides. In the present 2D system with TIs, the pseudospin-dependent one-way transmission is insensitive to arbitrary-shaped defects. From this point of view, such features cause the present acoustic TIs to possess unique advantages in guiding sound propagation in practical acoustic environment.

\section{CONCLUSIONS}

In this paper, we have theoretically demonstrated an approach to construct a type of $2 \mathrm{D}$ acoustic TI by projecting a section of a $3 \mathrm{D}$ twisting structure. This $2 \mathrm{D}$ acoustic TI model can be achieved by creating resonators with a higher refractive index than the ambient medium, such as perforated air holes in polished stainless-steel plates, aerogel as a soft acoustic metamaterial in air, and air in water. By calculating the acoustic band structures, pressure field distributions, and spin Chern numbers of bands, we have shown that the topological phases can mimic pseudospin-up and pseudospin-down states, and the pseudospin-orbital couplings can be tuned by changing the rotation angles of the meta-atoms, which eventually induce band inversion. In addition, we have illustrated that robust pseudospin-dependent one-way transmission is immune to the scattering of various types of defects at the edge between topologically distinct regions, which has potential applications in manipulating sound waves.

\section{ACKNOWLEDGMENTS}

This work was supported by the National Key R\&D Program of China (2017YFA0303702), by the National Natural Science Foundation of China (Grants No. 11634005, No. 11674155, No. 11604143, and No. 11621091), and partially by the "333 project" from Jiangsu Province (BRA2016350). Y.W. was supported by the King Abdullah University of Science and Technology (KAUST) Office of Sponsored Research (OSR-2016-CRG5-2950).
[1] R. B. Laughlin, Phys. Rev. Lett. 50, 1395 (1983).

[2] K. V. Klitzing, G. Dorda, and M. Pepper, Phys. Rev. Lett. 45, 494 (1980).

[3] C. L. Kane and E. J. Mele, Phys. Rev. Lett. 95, 226801 (2005).

[4] B. A. Bernevig, T. L. Hughes, and S. C. Zhang, Science 314, 1757 (2006).

[5] M. Z. Hasan and C. L. Kane, Rev. Mod. Phys. 82, 3045 (2010).

[6] X. L. Qi and S. C. Zhang, Rev. Mod. Phys. 83, 1057 (2011).

[7] L. Fu, Phys. Rev. Lett. 106, 106802 (2011).

[8] L. Lu, J. D. Joannopoulos, and M. Soljačić, Nat. Photonics 8, 821 (2014).

[9] L. H. Wu and X. Hu, Phys. Rev. Lett. 114, 223901 (2015).

[10] W. J. Chen, S. J. Jiang, X. D. Chen, B. Zhu, L. Zhou, J. W. Dong, and C. T. Chan, Nat. Commun. 5, 5782 (2014).

[11] X. D. Chen, Z. L. Deng, W. J. Chen, J. R. Wang, and J. W. Dong, Phys. Rev. B 92, 014210 (2015).

[12] M. Hafezi, S. Mittal, J. Fan, A. Migdall, and J. M. Taylor, Nat. Photonics 7, 1001 (2013).

[13] A. B. Khanikaev, S. H. Mousavi, W. K. Tse, M. Kargarian, A. H. MacDonald, and G. Shvets, Nat. Mater. 12, 233 (2013).

[14] F. Gao, Z. Gao, X. Shi, Z. Yang, X. Lin, H. Xu, J. D. Joannopoulos, M. Soljačić, H. Chen, L. Lu, Y. Chong, and B. Zhang, Nat. Commun. 7, 11619 (2016).

[15] B. Orazbayev, N. Kaina, and R. Fleury, Phys. Rev. Appl. 10, 054069 (2018)

[16] Q. Lin, X. Q. Sun, M. Xiao, S. C. Zhang, and S. Fan, Sci. Adv. 4, eaat2774 (2018).

[17] M. Xiao, Z. Q. Zhang, and C. T. Chan, Phys. Rev. X 4, 021017 (2014).

[18] J. Mei, Z. Chen, and Y. Wu, Sci. Rep. 6, 32752 (2016).

[19] M. Esmann, F. R. Lamberti, P. Senellart, I. Favero, O. Krebs, L. Lanco, C. Gomez Carbonell, A. Lemaître, and N. D. LanzillottiKimura, Phys. Rev. B 97, 155422 (2018).
[20] S. Y. Yu, C. He, Z. Wang, F. K. Liu, X. C. Sun, Z. Li, H. Z. Lu, M. H. Lu, X. P. Liu, and Y. F. Chen, Nat. Commun. 9, 3072 (2018).

[21] Z. Zhang, Q. Wei, Y. Cheng, T. Zhang, D. Wu, and X. Liu, Phys. Rev. Lett. 118, 084303 (2017).

[22] C. He, X. Ni, H. Ge, X. C. Sun, Y. B. Chen, M. H. Lu, X. P. Liu, and Y. F. Chen, Nat. Phys. 12, 1124 (2016).

[23] D. Torrent and J. Sanchez-Dehesa, Phys. Rev. Lett. 108, 174301 (2012).

[24] B. Z. Xia, T. T. Liu, G. L. Huang, H. Q. Dai, J. R. Jiao, X. G. Zang, D. J. Yu, S. J. Zheng, and J. Liu, Phys. Rev. B 96, 094106 (2017).

[25] Z. Yang, F. Gao, X. Shi, X. Lin, Z. Gao, Y. Chong, and B. Zhang, Phys. Rev. Lett. 114, 114301 (2015).

[26] Z. G. Chen and Y. Wu, Phys. Rev. Appl. 5, 054021 (2016).

[27] R. Fleury, D. L. Sounas, C. F. Sieck, M. R. Haberman, and A. Alù, Science 343, 516 (2014).

[28] Y. G. Peng, C. Z. Qin, D. G. Zhao, Y. X. Shen, X. Y. Xu, M. Bao, H. Jia, and X. F. Zhu, Nat. Commun. 7, 13368 (2016).

[29] C. He, Z. Li, X. Ni, X. C. Sun, S. Y. Yu, M. H. Lu, X. P. Liu, and Y. F. Chen, Appl. Phys. Lett. 108, 031904 (2016).

[30] Q. Wei, Y. Tian, S. Y. Zuo, Y. Cheng, and X. J. Liu, Phys. Rev. B 95, 094305 (2017).

[31] Y. Liu, C. S. Lian, Y. Li, Y. Xu, and W. Duan, Phys. Rev. Lett. 119, 255901 (2017).

[32] L. M. Nash, D. Kleckner, A. Read, V. Vitelli, A. M. Turner, and W. T. M. Irvine, Proc. Natl. Acad. Sci. USA 112, 14495 (2015).

[33] R. Süsstrunk and S. D. Huber, Science 349, 47 (2015).

[34] E. Prodan and C. Prodan, Phys. Rev. Lett. 103, 248101 (2009).

[35] T. Frenzel, M. Kadic, and M. Wegener, Science 358, 1072 (2017).

[36] R. Beravat, G. K. L. Wong, M. H. Frosz, X. M. Xi, and P. St. J. Russell, Sci. Adv. 2, e1601421 (2016). 
[37] K. Y. Bliokh, A. Niv, V. Kleiner, and E. Hasman, Nat. Photonics 2, 748 (2008).

[38] S. B. Wang, G. Ma, and C. T. Chan, Sci. Adv. 4, eaaq1475 (2018).

[39] M. Xiao, W. J. Chen, W. Y. He, and C. T. Chan, Nat. Phys. 11, 920 (2015).

[40] F. Li, X. Huang, J. Lu, J. Ma, and Z. Liu, Nat. Phys. 14, 30 (2017).

[41] J. Noh, S. Huang, D. Leykam, Y. D. Chong, K. P. Chen, and M. C. Rechtsman, Nat. Phys. 13, 611 (2017).

[42] L. Lu, C. Fang, L. Fu, S. G. Johnson, J. D. Joannopoulos, and M. Soljačić, Nat. Phys. 12, 337 (2016).

[43] B. Yang, Q. Guo, B. Tremain, R. Liu, L. E. Barr, Q. Yan, W. Gao, H. Liu, Y. Xiang, J. Chen, C. Fang, A. Hibbins, L. Lu, and S. Zhang, Science 359, 1013 (2018).

[44] H. X. Wang, L. Xu, H. Y. Chen, and J.-H. Jiang, Phys. Rev. B 93, 235155 (2016).
[45] M. C. Rechtsman, J. M. Zeuner, and Y. Lumer, Y. Plotnik, D. Podolsky, F. Dreisow, S. Nolte, M. Segev, and A. Szameit, Nature (London) 496, 196 (2013).

[46] M. D. Guild, V. M. Garcia-Chocano, J. Sanchez-Dehesa, T. P. Martin, D. C. Calvo, and G. J. Orris, Phys. Rev. Appl. 5, 034012 (2016).

[47] B. Liang, X. S. Guo, J. Tu, D. Zhang, and J. C. Cheng, Nat. Mater. 9, 989 (2010).

[48] A. Bretagne, A. Tourin, and V. Leroy, Appl. Phys. Lett. 99, 221906 (2011).

[49] V. Leroy, A. Bretagne, M. Fink, H. Willaime, P. Tabeling, and A. Tourin, Appl. Phys. Lett. 95, 171904 (2009).

[50] G. V. Frisk, Ocean and Seabed Acoustics: A Theory of Wave Propagation (Prentice-Hall, Englewood Cliffs, NJ, 1994).

[51] M. S. Dresselhaus, G. Dresselhaus, A. Jorio, and V. Heine, Group Theory: Application to the Physics of Condensed Matter (Springer-Verlag, Berlin, Heidelberg, 2008).

[52] T. Fukui, Y. Hatsugai, and H. Suzuki, J. Phys. Soc. Jpn. 74, 1674 (2005). 